\title{
THE STEADY MOTION OF A SYMMETRIC, FINITE CORE SIZE, COUNTERROTATING VORTEX PAIR*
}

\author{
JOSEPH YANG ${ }^{\dagger}$ AND TOSHI KUBOTA
}

\begin{abstract}
The steady motion of a symmetric, finite core size, counterrotating vortex pair is characterized by circulation $\Gamma$, a velocity $V$, and a spacing $2 x_{\infty}$. In the classical limit of a point vortex, the normalized velocity, $V x_{x} / \Gamma$, is $1 /(4 \pi)$. The effect of finite core size is to reduce the normalized velocity below the value for a point vortex. The flow is governed by a single geometrical parameter $R / x_{\infty}$, the ratio of effective vortex size to vortex half-spacing. Perturbation analysis is used to derive general, closed-form analytical solutions for the complete velocity field, the vortex pair velocity, and the boundary shape for a continuum of values of $R / x_{\infty}$. Both uniform and piecewise constant density cases are treated. These solutions illustrate the different orders at which the solution deviates from the point vortex pair. For example, the vortex shape becomes noncircular at order $\left(R / x_{\infty}\right)^{2}$, but the normalized velocity does not change until order $\left(R / x_{\infty}\right)^{5}$. For the uniform density case, calculation of specific values of vortex pair velocity, aspect ratio, and gap ratio shows good agreement with previous numerical results.
\end{abstract}

Key words. vortex pair, perturbation analysis, normalized velocity, boundary shape, steady state

AMS subject classifications. 76C05, 35Q30, 35B20

1. Introduction. Real vortex pair flows are often inadequately characterized by the classical point vortex pair model. A more realistic model considers the steady motion of a symmetric, finite core size, counterrotating vortex pair in an unbounded domain (Fig. 1). The vortices are characterized by a circulation $\Gamma$, a centroid-tocentroid spacing $2 x_{\infty}$, and a translational velocity $\mathrm{V}$. The area of either vortex is $A$, corresponding to an effective radius $R \equiv(A / \pi)^{1 / 2}$. The vorticity inside the vortices is uniform and equal to $\omega=\Gamma / A$, and the vorticity outside the vortices is zero. The density is piecewise constant inside and outside. The vortex pair moves steadily without change of area or shape.

In the case of finite core size, the normalized velocity $V x_{\infty} / \Gamma$ is reduced below the classical point vortex pair limit of $1 /(4 \pi)$. This is manifested by changes in the flow field and geometry of the vortex pair. We seek a family of vortices characterized by the single parameter $R / x_{\infty}$, the ratio of effective vortex size to vortex half-spacing.

Several investigators have treated the case of uniform density flow. Deem and Zabusky [1] used the Poisson equation for the stream function and vorticity, together with the appropriate boundary conditions for steady translational motion without change of boundary shape, to derive a nonlinear integrodifferential equation for the boundary shape. They solved this equation numerically with a Newton-Raphson method and discovered one vortex pair of fixed size, shape, and spacing that satisfied the governing equations.

* Received by the editors November 30, 1992; accepted for publication (in revised form) March 16, 1993. This work was supported by U.S. Air Force Office of Scientific Research contract F49620-86-C-0113 and grant AFOSR-90-0188. The first author was supported by an Office of Naval Research Graduate Fellowship. 91125.

${ }^{\dagger}$ Department of Mechanical Engineering, California Institute of Technology, Pasadena, California

${ }^{\ddagger}$ Department of Aeronantics, California Institute of Technology, Pasadena, California 91125. 


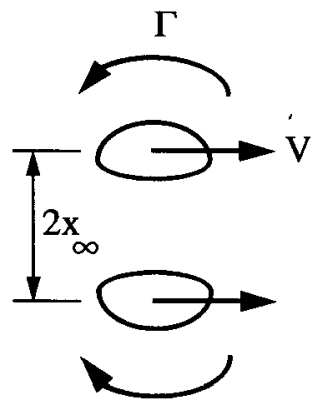

FIG. 1. Perturbation analysis in an unbounded domain.

Pierrehumbert [6] generalized the work of Deem and Zabusky to exhibit a family of solutions characterized by $R / x_{\infty}$. His family of solutions is shown in Fig. 2. Note that, as $R / x_{\infty}$ increases, the vortices become progressively less circular in shape. Only half of each vortex pair is plotted, the configuration being symmetric about the straight line corresponding to the flattened lower face of the outermost vortex core. Each member of the family has been scaled such that its outermost edge is at the same fixed distance from the line of symmetry. As $R / x_{\infty}$ increases, the velocity decreases monotonically from the constant, point vortex pair value.

"The decrease... may be traced to the elongation of the vortices as they approach each other. Essentially, when all the vorticity is concentrated in a small region, the velocity induced at a given point on a vortex by its image receives nearly the same contribution from each point on the image. When the vortices are elongated, however, the contribution from the distant portions of the image vortex are significantly less than those from the near portions, and the total induced velocity is correspondingly less than if the same circulation were concentrated in a small region" $[6$, p. 140].

As shown in the figure, Pierrehumbert originally proposed that the outermost contour intersects the line of symmetry between the two vortices as a cusp. Saffman and Szeto [9] and Saffman and Tanveer [10] argued analytically that the intersection was a corner rather than a cusp. Based on Saffman's results, Pierrehumbert [7] noted difficulties with the numerical procedure at the intersection and acknowledged the possibility of a corner. Wu, Overman, and Zabusky [11] numerically verified the expectation of a corner and also confirmed Pierrehumbert's results for the inner

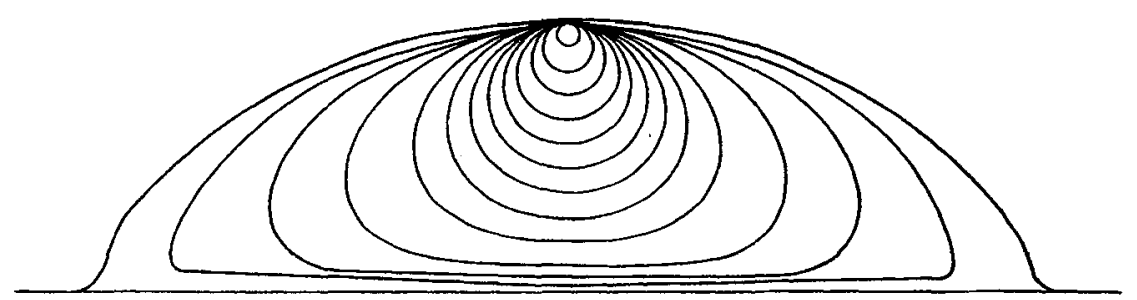

FIG. 2. Family of finite core size vortex pairs in an unbounded domain (reproduced from [6, p. 136]. Starting from the inside and moving outward, the contours correspond to $R / x_{\infty}=0.048,0.100,0.159,0.225$, $0.390,0.500,0.639,0.844,1.22,1.55,1.97$, and 2.16 . The outermost contour, which is the limit of touching vortices, is incorrect. 
members of this family. In a companion paper, Overman [5] presented yet another analytical argument for the corner.

This completed the numerical investigations of the uniform density flow. The solutions consisted of nonlinear integrodifferential equations, appropriate computational algorithms, and tabulated boundary shapes and vortex pair velocities for specific values of $R / x_{\infty}$. The results suggested that rather severe deformations from the ideal point vortex pair could be accommodated in boundary shape with relatively little change in velocity, but did not quantify the relative orders at which these processes occur. This is best determined analytically by means of an expansion in powers of some characteristic parameter. A solution consisting of closed-form, general expressions for the complete velocity field, boundary shapes, and vortex pair velocity for a continuum of values of $R / x_{\infty}$ would also offer distinct advantages in completeness and ease of use.

Melander, Styczek, and Zabusky [4] attempted such an analysis using a stream function formulation involving the integral of Biot-Savart contributions from differential elements of vorticity. Using the ratio of vortex area to the square of vortex spacing as an expansion parameter, they argued that the vortices were, to lowest order, ellipses. Comparison of their model with the numerical results showed good

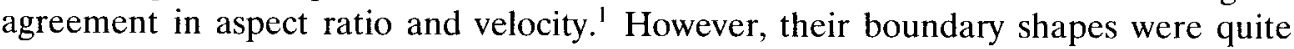
different, having two axes of symmetry instead of only one, as in the flattened teardrop shape of the numerical solutions. This indirectly verifies Saffman's [8] finding that an elliptical vortex pair is often a good approximation to the family of vortex pair flows described here.

We show below that the correct asymptotic expansion involves integral powers of the ratio of vortex size to vortex spacing. Melander, Styczek, and Zabusky, in choosing the square of this ratio as their expansion parameter, inadvertently discarded all the odd powers in the sequence. Instead of a stream function/Biot-Savart technique, we express the velocity as an analytic function of the complex spatial coordinate, i.e., a lowest-order point vortex solution with a Laurent series expansion of singularities located at both vortex centroids. The unknown coefficients in the expansion are determined by matching of orders, subject to appropriate dynamical constraints. Specific numerical values are compared to available numerical results. Finally, the solution for the piecewise constant density case is shown to be a straightforward transformation of that for the uniform density case.

\section{Perturbation solution}

2.1. Uniform density case. Consider first the case of uniform density everywhere. The area $A\left(=\pi R^{2}\right)$, vorticity $\omega(=\Gamma / A)$, and centroidal spacing $2 x_{\infty}$ of the vortex pair are specified. We seek expressions for the flow field and vortex shape as functions of a perturbation parameter $\varepsilon \equiv R / x_{x}$. The perturbation is shown in Fig. 3 . It is convenient to use a reference frame moving with the vortex pair, so that the vortices are not moving and the flow is steady. In this frame, the flow approaches from $y=+\infty$ with velocity $-V$. We take the line joining the centroids as the $x$-axis and put the origin at the centroid of one vortex; the other centroid is at $\left(-2 x_{\infty}, 0\right)$.

Vortex boundary. The boundary of the vortex located at the origin is expressed as

$$
r=R_{B}(\theta)
$$

\footnotetext{
${ }^{1}$ Values listed for their parameter $\varepsilon_{12}$ in their Table II are actually $2 \pi \varepsilon_{12}$.
} 


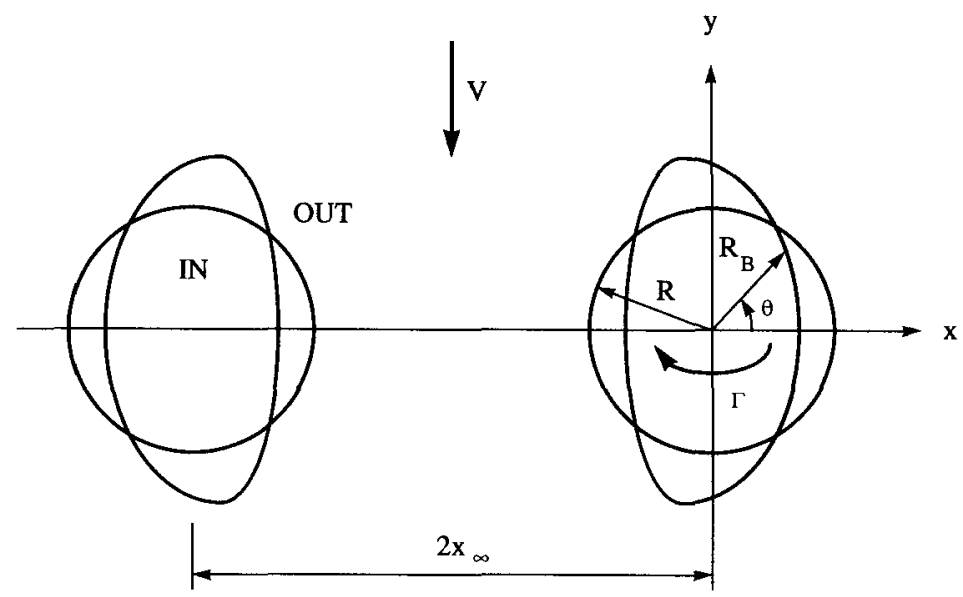

FIG. 3. Boundary shape schematic.

where $r$ and $\theta$ are the polar coordinates defined by

$$
r=\left(x^{2}+y^{2}\right)^{1 / 2}, \quad \tan \theta=\frac{y}{x} .
$$

Vortex centroid. Since the centroid is at the origin, we must have

$$
0=\iint x d A=2 \int_{0}^{\pi} d \theta \int_{0}^{R_{B}(\theta)}(r \cos \theta) r d r=\frac{2}{3} \int_{0}^{\pi} R_{B}^{3}(\theta) \cos \theta d \theta .
$$

Velocity fields. The velocity fields are conveniently expressed in terms of complex variables $z=x+i y$ and $w=u-i v$. It is necessary to distinguish between velocity fields inside and outside the vortices.

The velocity field outside is irrotational, and, by symmetry, $u=0$ along the $x$-axis $z=x$ and the line $z=-x_{\infty}+i y$. It may be expanded in the following series:

$$
w_{\text {out }}(z)=i V+\frac{i \Gamma}{\pi R} \sum_{n=1}^{\infty} a_{n}\left\{\left(\frac{R}{z}\right)^{n}+\left(\frac{-R}{z+2 x_{\infty}}\right)^{n}\right\},
$$

where the $a_{n}$ 's are real constants and $a_{1}=1$ because it represents a point vortex solution.

The velocity inside each vortex is given by the sum of a particular solution with the constant vorticity $\omega$, the velocity induced by the other vortex, and an irrotational flow. We need only consider one vortex, because the velocity field for the other vortex can be obtained by symmetry. For the vortex at the origin,

$$
w_{\text {in }}(z)=\frac{1}{2} i \omega \bar{z}+\frac{i \Gamma}{\pi R} \sum_{n=1}^{\infty} a_{n}\left(\frac{-R}{z+2 x_{\infty}}\right)^{n}+\frac{i \Gamma}{\pi R} \sum_{n=0}^{\infty} b_{n}\left(\frac{z}{R}\right)^{n} .
$$

Boundary conditions. The velocities $w_{\text {in }}$ and $w_{\text {out }}$ must satisfy the following matching conditions at the vortex boundary $r=R_{B}(\theta ; \varepsilon)$ :

1. The velocity component normal to the boundary is zero

$$
u_{n}=0
$$


where the velocity components normal and tangent to the boundary are given by

$$
u_{n}-i u_{t}=\frac{(u-i v)\left(R_{B}-i R_{B}^{\prime}\right) e^{i \theta}}{\left(R_{B}^{2}+R_{B}^{\prime 2}\right)^{1 / 2}}=\frac{-i w z_{B}^{\prime}}{\left(R_{B}^{2}+R_{B}^{\prime 2}\right)^{1 / 2}}
$$

with

$$
z_{B}=R_{B}(\theta) e^{i \theta}
$$

2. The pressure is continuous

$$
\rho_{\text {out }} u_{\text {out }}^{2}-\rho_{\text {in }} u_{\text {in }}^{2}=p_{t, \text { out }}-p_{t, \text { in }},
$$

where $p_{t, \text { out }}-p_{t, \text { in }}$ is the total pressure jump at the vortex boundary.

If

$$
p_{p, \text { in }}=p_{t, \text { out }}
$$

and

$$
\rho_{\text {in }}=\rho_{\text {out }},
$$

then

$$
u_{\text {in }}=u_{\text {out }} .
$$

Small $\varepsilon$ expansion. The boundary shape is expanded in terms of $\varepsilon$ as follows:

$$
R_{B}(\theta)=R+\sum_{k=1}^{\infty} \varepsilon^{k} r_{k}(\theta)
$$

The velocities inside and outside the vortex are also expanded in powers of $\varepsilon$; equivalently, the coefficients $a_{n}$ and $b_{n}$ in (4) and (5) are expanded as

$$
a_{n}=\sum_{k=1}^{\infty} q_{n}^{k} \varepsilon^{k}, \quad(n \geq 2)
$$

and

$$
b_{n}=\sum_{k=1}^{\infty} p_{n}^{k} \varepsilon^{k}, \quad(n \geq 0) .
$$

The vortex pair velocity is expressed as

$$
V=\varepsilon \pi \omega R\left(\frac{1}{4 \pi}+\sum_{k=1}^{\infty} \varepsilon^{k} V_{k}\right)
$$

or, since $\Gamma=\omega \pi R^{2}$,

$$
\frac{V x_{\infty}}{\Gamma}=\frac{1}{4 \pi}+\sum_{k=1}^{\infty} \varepsilon^{k} V_{k} .
$$

Substitute these expansions into (4) and (5) expressing the velocities outside and inside the boundary. The centroid equation (3), the normal velocity condition (6), and the matching condition (12) for the velocity components at the boundary give a sequence of equations for determining the coefficients $a_{n}$ and $b_{n}$, the vortex shape functions $r_{n}(\theta)$, and the vortex pair velocity $V$.

For each order of $\varepsilon$, the order of analysis is as follows. First, the inner and outer velocities are matched at the boundary. This expresses unknown coefficients $q_{n}^{k}$ and $r_{k}$ in terms of a common unknown $p_{n}^{k}$. Since the shape is symmetric about the $x$-axis, 
$R_{B}(\theta)$ must be an even function of $\theta$, and we expect the boundary shape coefficients $r_{k}$ to take the form of a Fourier cosine series with coefficients related to $p_{n}^{k}$. Next, - constancy of the centroidal spacing determines some of the unknown coefficients $p_{n}^{k}$. Finally, the normal velocity boundary condition determines the remainder of the unknown coefficients $p_{n}^{k}$ and the normalized velocity coefficients $V_{k}$. At this point, the solution is completely known to the specified order. Details of the order-by-order matching are shown in the Appendix. The complete velocity field may be found by substituting the coefficients listed there into (4), (5), (14), and (15). Expressions for boundary shape and translational velocity are summarized below. The shape is given by

$$
\begin{aligned}
\frac{R_{B}}{R}=1 & +\left(-\frac{1}{4} \cos 2 \theta\right) \varepsilon^{2}+\left(\frac{1}{16} \cos 3 \theta\right) \varepsilon^{3}+\left(-\frac{1}{64}+\frac{5}{192} \cos 4 \theta\right) \varepsilon^{4} \\
& +\left(\frac{1}{64} \cos \theta-\frac{3}{128} \cos 5 \theta\right) \varepsilon^{5} \\
& +\left(-\frac{1}{1024}+\frac{21}{512} \cos 2 \theta+\frac{77}{15360} \cos 6 \theta\right) \varepsilon^{6}+O\left(\varepsilon^{7}\right)
\end{aligned}
$$

the velocity is given by

$$
V=\pi \omega R\left[\left(\frac{1}{4 \pi}\right) \varepsilon-\left(\frac{1}{32 \pi}\right) \varepsilon^{5}\right]+O\left(\varepsilon^{7}\right),
$$

and the normalized velocity is given by

$$
\frac{V x_{\infty}}{\Gamma}=\frac{1}{4 \pi}-\left(\frac{1}{32 \pi}\right) \varepsilon^{4}+O\left(\varepsilon^{6}\right) .
$$

The boundary begins to deform $\varepsilon^{2}$, becoming elliptical in shape. At this order, there is no deviation from the point vortex pair velocity. At order $\varepsilon^{3}$, the boundary first exhibits a flattened teardrop shape, but there is still no change in velocity. Not until order $\varepsilon^{5}$ does the velocity deviate from the point vortex pair value. Carrying out the solution to sixth order shows no additional change in velocity, and the effort necessary for even higher orders is unjustified.

2.2. Comparison with previous results. We compare the analytical results against the numerical result as a check on the accuracy. The velocity is often the primary quantity of interest in vortical flows; because the sixth-order term contributes no additional accuracy, the results are only plotted to the fifth order. Figure 4(a) shows the family of boundary shapes from the perturbation analysis for $\varepsilon=0.048,0.100$, $0.159,0.225,0.390,0.500,0.639$, and 0.844 , corresponding to those of Fig. 2. The contours are plotted according to the convention used by Pierrehumbert, i.e., the extreme points of the boundary, normal to the direction of motion, are taken to be \pm 1 . Figure 4(b) shows the normalized velocity $V x_{\infty} / \Gamma$, which is also listed in Table 1 . Figure 4(c) shows Pierrehumbert's so-called "intervortex gap ratio," the ratio of the minimum and maximum spacings of corresponding points on the vortices' boundaries. Figure 4(d) shows the aspect ratio of either vortex. In all cases, for values of $R / x_{\infty}$ less than about 1.1, the results agree quite well. This both verifies the perturbation analysis and provides a check of the numerical analysis. 


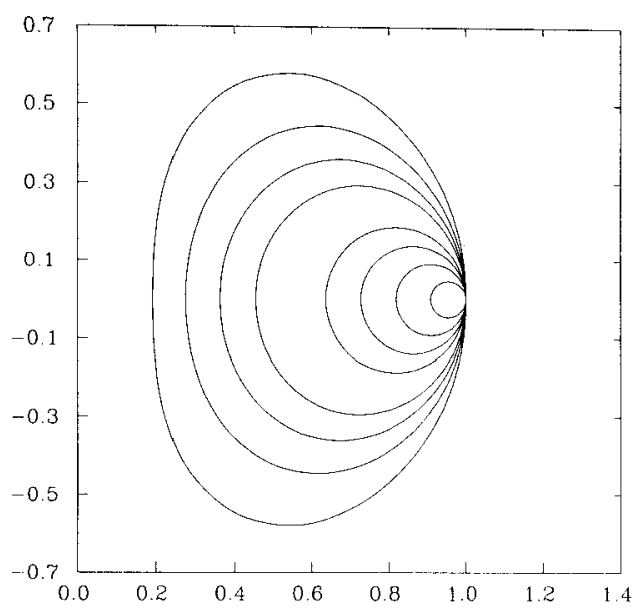

(a)

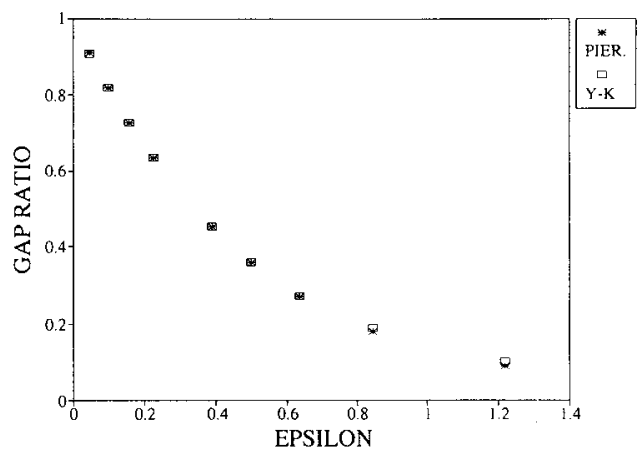

(c)

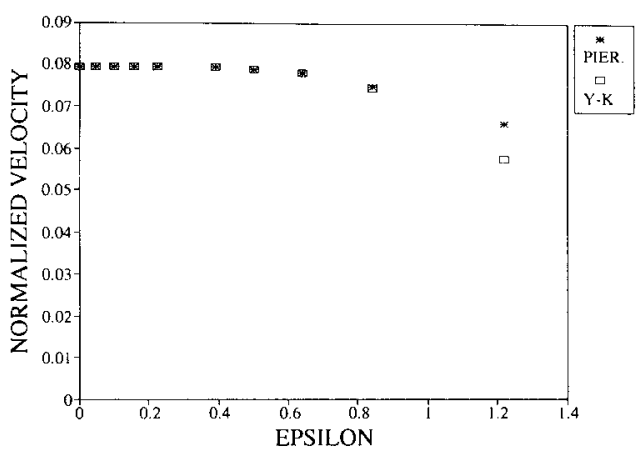

(b)

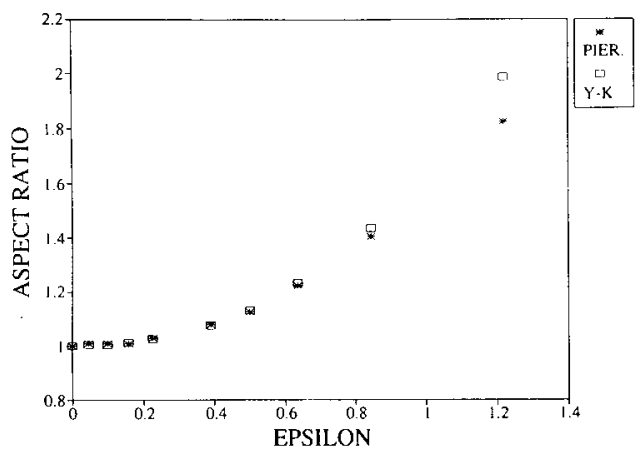

(d)

FIG. 4. Comparison of numerical and analytical results: (a) boundary shapes, (b) normalized velocities, (c) intervortex gap ratios, (d) aspect ratios.

TABLE 1

Normalized velocities.

\begin{tabular}{ccc}
\hline$\varepsilon$ & $\left(V x_{\infty} / \Gamma\right)_{Y K}$ & $\left(V x_{\infty} / \Gamma\right)_{P}$ \\
\hline 0.048 & 0.080 & 0.080 \\
0.100 & 0.080 & 0.080 \\
0.159 & 0.080 & 0.080 \\
0.225 & 0.080 & 0.080 \\
0.390 & 0.080 & 0.080 \\
0.500 & 0.079 & 0.079 \\
0.639 & 0.078 & 0.078 \\
0.844 & 0.075 & 0.075 \\
1.22 & 0.058 & 0.066 \\
\hline
\end{tabular}


The lowest-order solutions from Melander, Styczek, and Zabusky corresponding to (18)-(20) are given by

$$
\begin{gathered}
\frac{R_{B}}{R}=1+\left(-\frac{1}{4} \cos 2 \theta\right) \varepsilon^{2}+O\left(\varepsilon^{4}\right), \\
V=\pi \omega R\left[\left(\frac{1}{4 \pi}\right) \varepsilon-\left(\frac{1}{32 \pi}\right) \varepsilon^{5}\right]+O\left(\varepsilon^{7}\right),
\end{gathered}
$$

and

$$
\frac{V x_{x}}{\Gamma}=\frac{1}{4 \pi}-\left(\frac{1}{32 \pi}\right) \varepsilon^{4}+O\left(\varepsilon^{6}\right) .
$$

Although (21)-(23) are subsets of (18)-(20), they are inconsistent in orders of $\varepsilon$ (cf. order-by-order matching in the Appendix): this "lowest-order" solution gives powers of $\varepsilon^{2}$ in boundary shape, but powers of $\varepsilon^{4}$ in velocity. This indicates that $\varepsilon^{2}$ is not the proper expansion parameter. Note from (18) that it is the odd powers of $\varepsilon$ that contribute to a nonelliptical shape. The solutions represented by (21)-(23), even if continued to higher orders, would not be able to properly account for the deformation of boundary shape.

That $\varepsilon$ is the proper expansion parameter is also supported by Dhanak [2], who used an expansion in $\varepsilon$ to analyze the stability of a regular polygon of finite vortices. By extending this work to the case of a vortex pair, he can show agreement with (18) and (19) to the order given [3].

2.3. Piecewise constant density case. For convenience in the derivation of this solution, the density was assumed to be uniform everywhere. However, the uniform density solution can easily be transformed to the solution for the case of piecewise constant density.

Consider the governing equations for steady flow in the case of piecewise constant inner and outer densities. The inner flow continuity equation is

$$
\underline{\nabla} \cdot\left(\underline{u}_{\mathrm{in}}\right)=0,
$$

the inner flow momentum equation is

$$
\underline{\nabla} \cdot\left(\rho_{\text {in }} \underline{u}_{\text {in }} \underline{u}_{\text {in }}\right)+\underline{\nabla} p_{\text {in }}=0,
$$

the outer flow continuity equation is

$$
\underline{\nabla} \cdot\left(\underline{u}_{\text {out }}\right)=0,
$$

and the outer flow momentum equation is

$$
\underline{\nabla} \cdot\left(\rho_{\text {out }} \underline{u}_{\text {out }} \underline{u}_{\text {out }}\right)+\underline{\nabla} p_{\text {out }}=0 .
$$

We seek a relation between the inner and outer velocity fields. Defining a modified inner velocity as

$$
\underline{u}_{\text {in }}^{\prime} \equiv\left(\frac{\rho_{\text {in }}}{\rho_{\text {out }}}\right)^{1 / 2} \underline{u}_{\text {in }},
$$

equation (24) is transformed to

$$
\underline{\nabla} \cdot\left(\underline{u}_{\text {in }}^{\prime}\right)=0
$$


and (25) is transformed to

$$
\underline{\nabla} \cdot\left(\rho_{\text {out }} \underline{u}_{\text {in }}^{\prime} \underline{u}_{\text {in }}^{\prime}\right)+\underline{\nabla} p_{\text {in }}=0 .
$$

The density in these equations is now exactly the same as in the outer continuity and momentum equations. Thus, (26), (27), (29), and (30) exactly describe an equivalent uniform density flow, where inner velocities for the uniform density flow are interpreted as given by (28). Equivalently, the equations for uniform density flow exactly describe a piecewise constant density flow, provided that the velocities for the piecewise constant density flow are taken to be $\left(\rho_{\text {out }} / \rho_{\text {in }}\right)^{1 / 2}$ times those determined for the uniform density flow.

It is also necessary to check the boundary conditions for the uniform density flow to see that the appropriate boundary condition is satisfied for the piecewise constant density flow. The total pressures inside and outside the vortices are again assumed to be the same (10). Then the first boundary condition, continuity of velocity in the uniform density flow (12), correctly implies continuity of pressure (9) in the piecewise constant density flow under the velocity transformation (28). That is, velocity matching in the uniform density flow automatically satisfies pressure matching in the piecewise constant density flow. The second boundary condition, that the surface velocity be tangential to the boundary (6), is the same in either case.

Thus the perturbation analysis, which was carried out for a uniform density flow, is directly applicable to a piecewise constant density flow. The outer velocities, boundary shapes, and normalized velocities are the same in either case. The inner velocities for the piecewise constant density flow are simply constant multiples of the inner velocities found for the uniform density case.

3. Conclusions. The steady motion of a symmetric, finite core size, counterrotating vortex pair in an unbounded domain was solved via a perturbation analysis. Closed-form, analytical expressions were derived for the complete velocity field, boundary shape, and translational velocity for a continuum of values of $R / x_{\infty}$. The family of allowable solutions is parameterized by a single parameter $R / x_{\infty}$, the ratio of effective vortex size to vortex half-spacing. As $R / x_{\infty}$ increases from zero, the vortices become progressively more flattened perpendicular to the direction of motion, and their translational velocity decreases below the classical point vortex limit. Both uniform and piecewise constant density cases were treated. Compared to the constant density flow, the piecewise constant solution has the same boundary shape, outer velocity field, and translational velocity as the constant density flow. The inner velocity field is simply a constant multiple of that for the constant density flow.

One advantage of an analytical solution over a numerical solution is its illustration of the physics of the flow. For example, it is found that the boundary shape begins to deform from an unperturbed circular shape at order $O\left(R / x_{\infty}\right)^{2}$, but the velocity remains constant until order $O\left(R / x_{\infty}\right)^{5}$. Other advantages include completeness (e.g., determination of the complete velocity field) and ease of use (e.g., availability of a continuum of values of $R / x_{\infty}$ as opposed to a finite set of tabulated values).

For the specific case of a uniform density flow, numerical values computed using the analytical expressions show good quantitative agreement with Pierrehumbert's numerical solution for values of $R / x_{\infty}$ less than about 1.1. A previous analytical solution showed good translational velocity agreement with the numerical results, but cannot predict the boundary shapes because the odd terms in the correct asymptotic expansion were omitted. 
Appendix. (i) To order $\varepsilon^{0}$ : All conditions are identically satisfied.

(ii) To order $\varepsilon^{1}$ : Velocity matching gives

$$
q_{n+1}^{1}=-p_{n-1}^{1}, \quad(n \geq 1)
$$

and

$$
r_{1}=-\frac{2}{\omega} \sum_{n=1}^{\infty} p_{n-1}^{1} \cos n \theta
$$

the centroid condition gives $p_{0}^{1}=0$; and the normal velocity condition gives $p_{n}^{1}=0$, $(n \geq 1)$. After substitution, we find that $r_{1}=0$.

(iii) To order $\varepsilon^{2}$ : Velocity matching gives

$$
q_{n+1}^{2}=-p_{n-1}^{2}, \quad(n \geq 1)
$$

and

$$
r_{2}=-\frac{2}{\omega} \sum_{n=1}^{\infty} p_{n-1}^{2} \cos n \theta
$$

the centroid condition gives $p_{0}^{2}=0$; and the normal velocity condition gives

$$
V_{1}=0, \quad p_{1}^{2}=\frac{1}{8} \omega R,
$$

and

$$
p_{n}^{2}=0, \quad(n \geq 2) .
$$

After substitution, we find that $r_{2}=-\frac{1}{4} R \cos 2 \theta$.

(iv) To order $\varepsilon^{3}$ : Velocity matching gives

$$
q_{n+1}^{3}=-p_{n-1}^{3}, \quad(n \geq 1)
$$

and

$$
r_{3}=-\frac{2}{\omega} \sum_{n=1}^{\infty} p_{n-1}^{3} \cos n \theta ;
$$

the centroid condition gives $p_{0}^{3}=0$; and the normal velocity condition gives

$$
V_{2}=0, \quad p_{2}^{3}=-\frac{1}{32} \omega R,
$$

and

$$
p_{n}^{3}=0, \quad(n \neq 2) .
$$

After substitution, we find that $r_{3}=\frac{1}{16} R \cos 3 \theta$.

(v) To order $\varepsilon^{4}$ : Velocity matching gives

$$
q_{5}^{4}=-p_{3}^{4}+\frac{1}{16} \omega R
$$

and

$$
q_{n+1}^{4}=-p_{n-1}^{4}, \quad(n \neq 4)
$$

$u_{\theta}$-matching gives

$$
r_{4}=-\frac{1}{64} R+\frac{3}{64} R \cos 4 \theta-\frac{2}{\omega} \sum_{n=1}^{\infty} p_{n-1}^{4} \cos n \theta
$$


the centroid condition gives $p_{0}^{4}=0$; and the normal velocity condition gives

and

$$
V_{3}=0, \quad p_{3}^{4}=\frac{1}{96} \omega R,
$$

$$
p_{n}^{4}=0, \quad(n \neq 3) .
$$

After substitution, we find that

$$
r_{4}=-\frac{1}{64} R+\frac{5}{192} R \cos 4 \theta .
$$

(vi) To order $\varepsilon^{5}$ : Velocity matching gives

$$
q_{2}^{5}=-p_{0}^{5}-\frac{1}{128} \omega R, \quad q_{6}^{5}=-p_{4}^{5}-\frac{5}{128} \omega R,
$$

and

$$
q_{n+1}^{5}=-p_{n-1}^{5}, \quad(n \neq 1,5)
$$

$u_{\theta}$-matching gives

$$
r_{5}=-\frac{1}{32} R \cos 5 \theta-\frac{2}{\omega} \sum_{n=1}^{\infty} p_{n-1}^{5} \cos n \theta ;
$$

the centroid condition gives $p_{0}^{5}=-\frac{1}{128} \omega R$; and the normal velocity condition gives

$$
V_{4}=-\frac{1}{32 \pi}, \quad p_{4}^{5}=-\frac{1}{256} \omega R
$$

and

$$
p_{n}^{5}=0, \quad(n \neq 0,4) .
$$

After substitution, we find that

$$
r_{5}=\frac{1}{64} R \cos \theta-\frac{3}{128} R \cos 5 \theta .
$$

(vii) To order $\varepsilon^{6}$ : Velocity matching gives

$$
\begin{gathered}
q_{3}^{6}=-p_{1}^{6}-\frac{1}{384} \omega R, \\
q_{7}^{6}=-p_{5}^{6}-\frac{9}{512} \omega R, \\
q_{n+1}^{6}=-p_{n-1}^{6}, \quad(n \neq 2,6),
\end{gathered}
$$

and

$$
r_{6}=-\frac{1}{1024} R+\frac{7}{1536} R \cos 2 \theta+\frac{25}{3072} R \cos 6 \theta-\frac{2}{\omega} \sum_{n=1}^{\infty} p_{n-1}^{6} \cos n \theta ;
$$

the centroid condition gives $p_{0}^{6}=0$; and the normal velocity condition gives

$$
V_{5}=0, \quad p_{1}^{6}=-\frac{7}{384} \omega R, \quad p_{5}^{6}=\frac{1}{640} \omega R,
$$

and

$$
p_{n}^{6}=0, \quad(n \neq 1,5) .
$$

After substitution, we find that

$$
r_{6}=-\frac{1}{1024} R+\frac{21}{512} R \cos 2 \theta+\frac{77}{15360} R \cos 6 \theta .
$$




\section{REFERENCES}

[1.] "̆. S. DEEM AND N. J. ZABUSKY, Vortex waves: Stationary "V states," interactions, recurrence, and breaking, Phys. Rev. Lett., 40 (1978), pp. 859-862.

[2] M. R. DhanaK, Stability of a regular polygon of finite vortices, J. Fluid Mech., 234 (1992), pp. $297-316$.

[3] — - private communication, Florida Atlantic University, Boca Raton, FL, March 1993.

[4] M. V. MELANDER, A. S. STyczek, AND N. J. ZabuSky, Elliptically desingularized vortex model for the two-dimensional Euler equations, Phys. Rev. Lett., 53 (1984), pp. 1222-1225.

[5] E. A. Overman, Steady-state solutions of the Euler equations in two dimensions II, Local analysis of limiting $V$-states, SIAM J. Appl. Math., 46 (1986), pp. 765-800.

[6] R. T. Pierrehumbert, A family of steady, translating vortex pairs with distributed vorticity, J. Fluid Mech., 99 (1980), pp. 129-144.

[7] —_, Corrigendum to A family of steady, translating vortex pairs with distributed vorticity, J. Fluid Mech., 102 (1981), p. 479.

[8] P. G. SAFFman, The approach of a vortex pair to a plane surface in inviscid fluid, J. Fluid Mech., 92 (1979), pp. 497-503.

[9] P. SAFFMAN AND R. SZETO, Equilibrium shapes of a pair of equal uniform vortices, Phys. Fluids, 23 (1980), pp. 2339-2342.

[10] P. G. SaFfman And S. Tanveer, The touching pair of equal and opposite uniform vortices, Phys. Fluids, 25 (1982), pp. 1929-1930.

[11] H. M. Wu, E. A. OvermAN, AND N. J. ZABUSKY, Steady-state solutions of the Euler equations in two dimensions: Rotating and translating V-states with limiting cases. I. Numerical algorithms and results, J. Comput. Phys., 53 (1984), pp. 42-71. 